\title{
Coma blisters after a syncope episode
}

\author{
Gabriela Maloney, Xin Liu, Drew Taylor, Marylee Braniecki \\ Midwestern University, Chicago, United States \\ Correspondence: Gabriela Maloney. Address: Midwestern University, Chicago, United States. \\ Email: gabrielamaloney@gmail.com
}

Received: November 4, 2014

Accepted: November 23, 2014 Online Published: May 11, 2015

DOI : $10.5430 /$ crim.v2n3p5

URL: http://dx.doi.org/10.5430/crim.v2n3p5

\begin{abstract}
We report a case of a 74-year-old man who developed an acute blistering eruption on both extremities after a syncope episode (for undetermined amount of time) with altered mental status. Tense and denuded bullae were found on non-blanching erythematous to violaceous skin involving the left forearm, left dorsal hand, left interdigital spaces, left upper chest and right medial knee. His medical history included diabetes mellitus, hypertension, end stage renal disease and a renal transplant 4 years ago. Patient did not have a history of similar skin lesions in the past. Skin biopsy revealed the development of an intraepidermal acrosyringeal bulla, accompanied by superficial, non-inflammatory purpura and stromal edema demonstrating the early stage of a coma blister before the characteristic onset of eccrine gland necrosis. Coma blisters are self-limited cutaneous lesions characterized by tense bullae at sites of maximal pressure. They are diagnosed histologically by necrotic eccrine glands with minimal or absent inflammation. Although it occurs most commonly in the setting of coma with barbiturate overdose, they can be seen in coma due to other etiologies, or even occur in a conscious state. Possible complications may include non-traumatic rhabdomyolysis and compression neuropathies, and may vary depending on patient's cause of unconsciousness and underlying medical conditions. Coma blisters look clinically alarming but they tend to spontaneously heal without specific treatment other than positional changes. In critical care settings where complex comorbidities exist, it is important to remember that coma blisters do not represent a contraindication to continue any ongoing therapy.
\end{abstract}

\section{Keywords}

Coma blister, Bulla, Altered mental status, Pressure bulla

\section{Introduction}

Coma blisters are self-limited cutaneous lesions characterized by tense bullae at sites of maximal pressure. Although it occurs most commonly in the setting of coma with barbiturate overdose, they can be seen in coma due to other etiologies, or even occur in a conscious state.

\section{Case presentation}

We report a case of a 74-year-old man who developed an acute blistering eruption on both extremities after a syncope episode (for undetermined amount of time) with altered mental status. This patient was found lying on the floor of his home by his son. He was dehydrated and had severe hypernatremia. Tense and denuded bullae were found on 
non-blanching erythematous to violaceous skin involving the left forearm, left dorsal hand, left interdigital spaces, left upper chest and right medial knee. Bullae were negative for Nikolsky and Asboe-Hansen signs. Patient was also negative for milia and oral lesions. His medical history included diabetes mellitus, hypertension, end stage renal disease and a renal transplant 4 years ago. His medications included Prednisone, Tacrolimus, Amlodipine, Doxazosin, iron sulfate, Hydralazine, Metoprolol and phosphate binder. Patient did not have a history of similar skin lesions in the past. Skin biopsy revealed an intraepidermal blister without inflammation (see Figure 1).

Serial tissue sections of skin demonstrate the development of an intraepidermal acrosyringeal bulla, accompanied by superficial, non-inflammatory purpura and stromal edema (see Figure 2). One sees modest stromal RBC extravasation, mild capillary ectasia, mild stromal edema and hemosiderin deposition in the subcutaneous fat. The adjacent epidermis appears intact, without spongiosis, inflammation or ischemic changes.

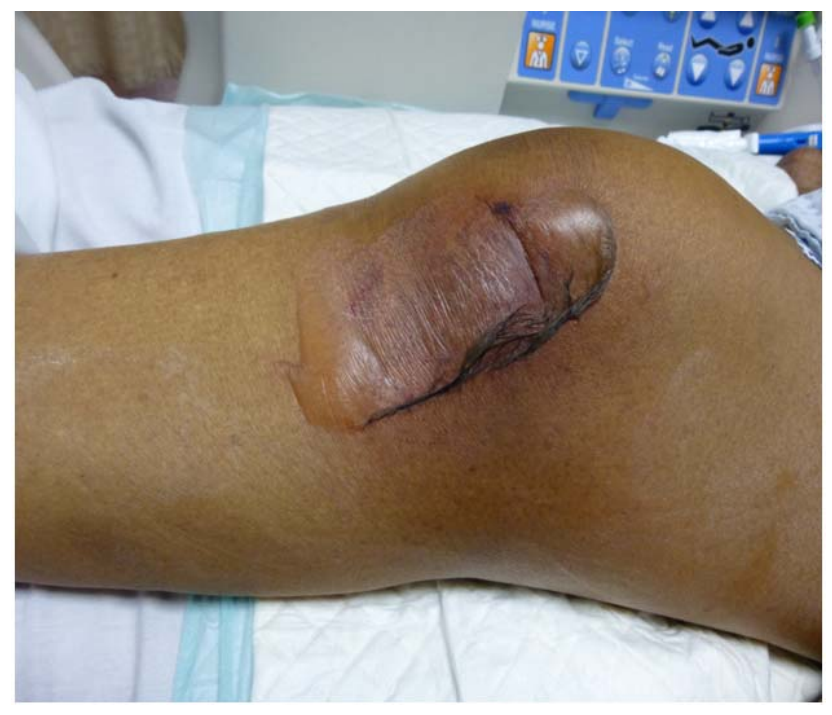

Figure 1. Tense bullae on a pressure site
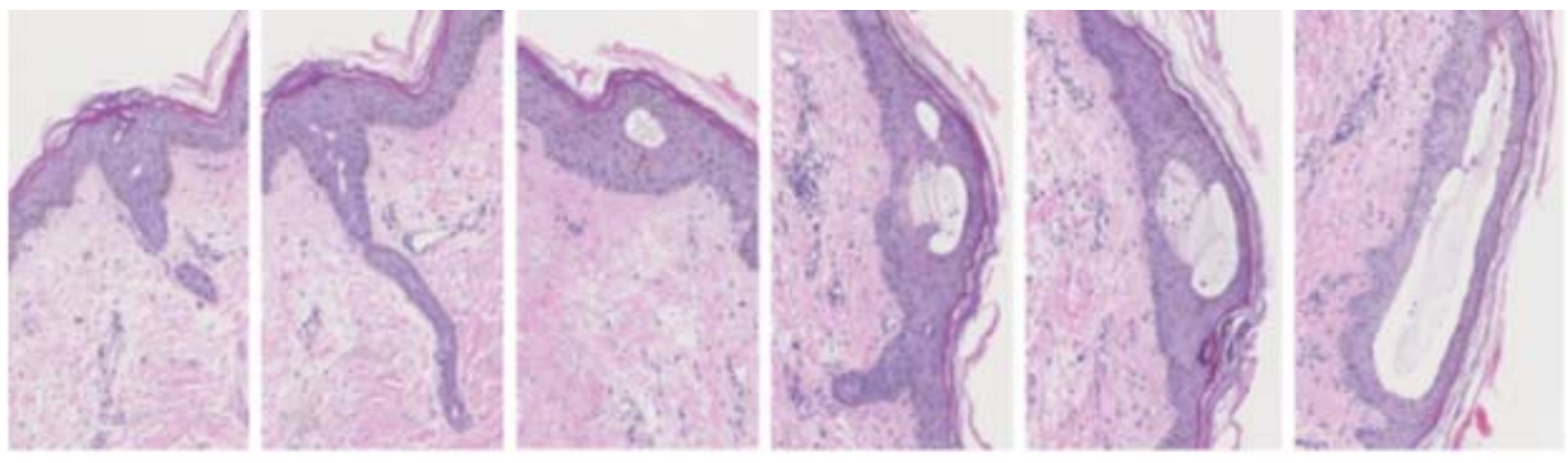

Figure 2. Serial skin sections show the early stages of coma blister: Development of a cell poor intra-epidermal blister associated with acrosyringeal dilatation before onset of eccrine gland necrosis

\section{Discussion}

Coma blisters clinically present as tense blisters and most commonly develop in coma patients at pressure sites after a prolonged period unconsciousness of at least 24 hours. This condition has a high association with coma resulting from drug overdoses such as barbiturates, opioids, tricyclic antidepressants, antipsychotics ${ }^{[1]}$ or benzodiazepines ${ }^{[2]}$. However, 
it has also been reported to occur in the settings of non-drug induced coma ${ }^{[3]}$ such as viral infections, encephalitis, diabetic ketoacidosis, carbon monoxide poisoning, trauma, cerebrovascular accidents, hepatic encephalopathy and hypoglycemia $^{[1]}$. Thus, it is important to acknowledge that loss of consciousness is not a requirement for the diagnosis of coma blisters.

The clinical course of the disease starts approximately 24 hours after the onset of coma with the appearance of patches or plaques of blanchable erythema. These patches then proceed to blisters or erosions within 48-72 hours of onset, which usually heal within one to two weeks with possible residual scaring. Possible complications may include non-traumatic rhabdomyolysis and compression neuropathies ${ }^{[1]}$, and may vary depending on patient's cause of unconsciousness and underlying medical conditions.

Although the exact pathogenesis of the blistering condition is unknown, it is believed that it is due to tissue hypoxia caused by the constant pressure ${ }^{[4]}$. It has been recognized that the eccrine gland is a metabolically active unit and appears to be very susceptible to ischemic necrosis. Thus, the histological observation of necrotic eccrine glands with minimal or absent inflammation is a characteristic finding of coma blisters ${ }^{[4]}$. Necrosis can also be present in the epithelium of pilosebaceous follicles. Direct immunofluorescence (DIF) may show IgM and C3 granular deposits around dermal blood vessels, but DIF is usually negative ${ }^{[2]}$.

Differential diagnosis may include friction blister, bullous diabeticorum, porphyria and acquired epidermolysis bullosa. A coma blister differs from a friction blister in that a friction blister typically shows blistering above the granular layer of the epidermis associated with surface irritative changes. Bullous diabeticorum usually does not show eccrine gland necrosis, but rather chronic ischemic atrophic skin changes, small vessel hyalinizing angiopathy and subepidermal blister formation. A blister caused by porphyria would also show a subepidermal blister accompanied by PAS (diastase resistant) vessel walls and thickened basement membrane with festooning. Acquired epidermolysis bullosa occurs in areas of trauma and shows evident immunodeposition on DIF study.

This is an interesting case of coma blister in that it histologically demonstrates the early stage of a coma blister before the characteristic onset of eccrine gland necrosis. Serial step sections of the skin biopsy show an intra-epidermal blister development accompanied by acrosyringeal dilatation.

\section{Conclusion}

Coma blisters tend to spontaneously heal, so no treatment is necessary other than positional changes. They may look clinically alarming but spontaneously heal without specific treatment other than positional changes. In critical care settings where complex comorbidities exist, it is important to remember that coma blisters do not represent a contraindication to continue any ongoing therapy ${ }^{[4]}$. Being aware of this entity can help manage complicated patients with various comorbidities.

\section{References}

[1] Bolognia JL, Jorizzo JL, Schaffer JV. Dermatology. Elsevier Saunders. 2012; 33: 515-521.

[2] Heinisch S, Loosemore M, Cusack C. Coma Blisters Sans Coma. Cutis. 2012; 90: 137-9. PMid:23094313

[3] Kato N, Ueno H, Mimura M. Histopathology of Cutaneous Changes of Non-Drug Induced Coma. Am J Dermatopathol. 1996; 18(4): 344-50. PMid:8879296 http://dx.doi.org/10.1097/00000372-199608000-00003

[4] Rocha J, Pereira T, Ventura F, et al. Coma Blisters. Case Rep in Dermatol. 2009; 1: 66-70. PMid:20652118 http://dx.doi.org/10.1159/000249150 